\title{
Stop-catalyzed baryogenesis beyond the MSSM
}

\author{
Andrey Katz \\ Theory Division, CERN, CH-1211 Geneva 23, Switzerland \\ and Université de Genève, Department of Theoretical Physics and Center for Astroparticle Physics (CAP), \\ 24 quai E. Ansermet, CH-1211 Geneva 4, Switzerland \\ Maxim Perelstein \\ Laboratory for Elementary Particle Physics, Cornell University, Ithaca, New York 14853, USA \\ Michael J. Ramsey-Musolf \\ Amherst Center for Fundamental Interactions, Department of Physics, \\ University of Massachusetts-Amherst Amherst, Massachusetts 01003, USA \\ and Kellogg Radiation Laboratory, California Institute of Technology, Pasadena, California 91125, USA
}

Peter Winslow

Amherst Center for Fundamental Interactions, Department of Physics, University of Massachusetts-Amherst Amherst, Massachusetts 01003, USA

(Received 21 September 2015; published 19 November 2015)

\begin{abstract}
Nonminimal supersymmetric models that predict a tree-level Higgs mass above the minimal supersymmetric standard model (MSSM) bound are well motivated by naturalness considerations. Indirect constraints on the stop sector parameters of such models are significantly relaxed compared to the MSSM; in particular, both stops can have weak-scale masses. We revisit the stop-catalyzed electroweak baryogenesis (EWB) scenario in this context. We find that the LHC measurements of the Higgs boson production and decay rates already rule out the possibility of stop-catalyzed EWB. We also introduce a gauge-invariant analysis framework that may generalize to other scenarios in which interactions outside the gauge sector drive the electroweak phase transition.
\end{abstract}

DOI: $10.1103 /$ PhysRevD.92.095019

PACS numbers: $12.60 . J v$

\section{INTRODUCTION}

The origin of matter-antimatter asymmetry in the Universe is a longstanding problem at the interface of particle and nuclear physics with cosmology. This issue cannot be addressed within the standard model (SM) and requires physics beyond the SM. One of the most interesting possibilities, which has attracted much attention in recent years, is the electroweak baryogenesis (EWB) scenario (see [1] for review), where the baryon asymmetry is produced during the electroweak phase transition (EWPT). This mechanism requires new physics beyond the standard model (BSM) at the weak scale $(\sim 100 \mathrm{GeV})$ for two different reasons:

(i) The SM with the Higgs boson mass above $\sim 80 \mathrm{GeV}$ does not satisfy the Sakharov criterion [2] of departure from thermal equilibrium because the EWPT is a crossover, rather than a strong 1st-order transition [3-6]. In order for EWB to be viable, one

Published by the American Physical Society under the terms of the Creative Commons Attribution 3.0 License. Further distribution of this work must maintain attribution to the author(s) and the published article's title, journal citation, and DOI. inevitably needs new bosonic fields that couple to the Higgs and significantly change the EWPT dynamics.

(ii) Although the $\mathrm{SM}$ does violate $C P$ symmetry, the effects are highly suppressed at temperatures $T \sim$ $100 \mathrm{GeV}$ by quark Yukawa couplings. Consequently, even if a SM universe admitted a strong 1st-order EWPT the produced baryon asymmetry is too small [7-9]. New sources of $C P$ violation at the weak scale are required.

Weak-scale supersymmetry (SUSY) is a well-motivated extension of the SM which can address both these problems. Electroweak gaugino/Higgsino $[10,11]$ and scalar [12] phases can provide the new sources of $C P$ violation (CPV), while the nature of EWPT can drastically change either in the presence of low-mass stops [13-15], or in models with extended Higgs sectors, e.g. additional singlet in the next-to-minimal supersymmetric standard model (NMSSM) [16-18]. In both cases, present and future experimental probes of BSM physics may provide conclusive tests. Present limits on the permanent electric dipole moments (EDMs) of the electron, neutron, and neutral atoms place stringent constraints on these $C P$-violating sources, and future EDM searches may probe the remaining CPV parameter space [19]. At the same time, searches for 
new scalar particles at the CERN Large Hadron Collider may uncover the ingredients needed for a first order EWPT.

In this paper, we focus on the possibility that low-mass stops may give rise to the first order EWPT, the so-called stop-catalyzed electroweak baryogenesis scenario. The basic idea is that a very light stop $(m \approx 100 \mathrm{GeV})$ modifies the Higgs potential at finite temperatures via quantum loop effects, inducing a barrier along the Higgs direction between the electroweak symmetric and broken vacua and triggering a strongly 1st-order EWPT. It has been already shown in $[20,21]$ that this scenario is no longer viable in the context of minimal supersymmetric standard model (MSSM), in light of the LHC measurements of the Higgs boson mass, production cross sections and decay rates (for pre-LHC works on the same subject see [22]).

In this work we analyze the stop-catalyzed baryogenesis in a more generic SUSY framework. Specifically, we consider adding small, hard-SUSY breaking terms to the Higgs potential. (Such terms can be dynamically generated at the few-TeV energy scale; for examples, see [23-26]). The primary motivation for models of this type comes from considerations of naturalness [27]. The new potential terms can give tree-level contributions to the SM-like Higgs mass, allowing for a SUSY theory with an SM-like Higgs at $125 \mathrm{GeV}$ independently of the stop sector parameters. Spectra with two relatively light stops can then still be viable, reducing fine-tuning. One may hope that the wideopen stop parameter space of these models may also allow them to accommodate the stop-catalyzed EWB scenario. This paper will explore whether this is indeed the case.

Unfortunately, we find that even in this broader framework, there is still no parameter space which is compatible with both the strong 1st order EWPT and the LHC Higgs measurements. The key observation which leads us to this conclusion is that the light stop can change the order of the phase transition only if its coupling to the Higgs is close to its maximal possible value, which occurs when the light stop is nearly a pure gauge eigenstate (that is, $\tilde{t}_{1} \approx \tilde{t}_{L}$ or $\left.\tilde{t}_{1} \approx \tilde{t}_{R}\right)$. On the other hand, in the small-mixing limit, loops of the light stop induce very large $(\sim 100 \%)$ shifts in the Higgs couplings to photons and gluons, in contradiction with the LHC data that requires these couplings to be within $10 \%-20 \%$ of their SM values. It has been proposed in Ref. [28] that this problem can be resolved if one assumes an appreciable invisible rate of the Higgs particle into neutralino pairs, which would compensate for the growth in $h g g$ coupling due to the light stop. We show that this modification is also no longer compatible with the LHC Higgs data. Therefore, we conclude that stop-catalyzed EW baryogenesis is excluded by data over the entire parameter space, even if the MSSM Higgs mass constraint is removed.

The paper is organized as follows. In Sec. II we briefly review the idea behind stop-catalyzed baryogenesis and describe in detail our framework of SUSY beyond MSSM. In Sec. III we review the existing experimental constraints on the light stops, including both direct LHC searches and indirect constraints from measurements of the Higgs properties. In Sec. IV we discuss the conditions for a strong 1st order EWPT with an emphasis on gaugeinvariance of the analysis. The framework that we introduce in this context may generalize to other scenarios in which new interactions outside the gauge sector drive the EWPT dynamics. Section V discusses some other technical details of our analysis, as well as its main results. Finally, the conclusions are summarized in Sec. VI.

\section{SUSY BARYOGENESIS AND SUSY BEYOND THE MSSM}

In the SM with the Higgs mass above $\gtrsim 80 \mathrm{GeV}$ the EWPT is a crossover [3-6]. Assuming that the phase transition occurs in a single step, any viable EWB scenario, thus, requires augmenting the Higgs sector with new bosonic degrees of freedom that couple strongly to the Higgs (for alternatives to the single-step phase transition scenario, see, e.g. Refs. [29-35]). One generic possibility is new scalar particles that generate a barrier between the electroweak symmetric and broken vacua in the Higgs thermal potential via quantum loops, leading to a strongly 1st order EWPT. SUSY provides a natural candidate for such a particle: the stop. The coupling of the stop to the Higgs is predicted by SUSY and it is equal (up to the trilinear term) to the top Yukawa squared, an order-one number. It has been shown in $[13,15,28]$ that light stops, with masses roughly in the $100 \ldots 120 \mathrm{GeV}$ range, can trigger a strongly 1st order EWPT, if the stops are not heavily mixed and the $\tan \beta$ is large, $\gtrsim 10$. The reason for this constraint is that mixing reduces the effective coupling between the stop and the SM-like Higgs. Most of the work on this scenario assumed that the light stop is purely righthanded, to avoid introducing a very light sbottom. In this paper, we do not make this assumption, considering instead the most general stop sector characterized by three parameters, the physical stop masses $m_{\tilde{t}_{1}}$ and $m_{\tilde{t}_{2}}$, and the rotation angle from gauge to mass eigenbasis, $\theta_{t}$. (In some of the plots, we will find it useful to trade $\theta_{t}$ for the mixing parameter $X_{t}$, defined as $X_{t} \equiv M_{L R}^{2} / m_{t}$.) Doing so will allow us to quantify the extent of mixing for which the strong 1st-order transition is possible, as well as to contrast the parameter space required by the EWB scenario with that allowed by the LHC data.

Discovery of the $125 \mathrm{GeV}$ Higgs disfavors the pure MSSM, which predicts $m_{h} \leq m_{Z}$ at tree level. The one-loop contribution to the Higgs mass-squared is given by

$$
\Delta m_{h}^{2}=\frac{3 y_{t}^{2}}{4 \pi^{2}} \cos ^{2} \alpha m_{t}^{2} \log \left(\frac{m_{\tilde{t}_{1}} m_{\tilde{t}_{2}}}{m_{t}^{2}}\right)+\cdots
$$

where the ellipsis stand for the terms proportional to the Higgs mixing and higher-loop corrections [36-38]. Very high stop masses are required to accommodate the 
measured Higgs mass. For example, if $m_{\tilde{t}_{1}} \approx 100 \mathrm{GeV}$, as required in the stop-catalyzed EWB scenario, the second stop would need to have a mass of order $100 \mathrm{TeV}$ to $\mathrm{PeV}$, unless $X_{t} \gtrsim 1 \mathrm{TeV}$ [39]. Such a spectrum would imply significant fine-tuning in the electroweak scale. In addition, the hierarchy of three or more orders of magnitude between the left-handed and right-handed squark soft masses would be challenging to explain from a model-building point of view. Finally, even if these issues are ignored, the purelyMSSM version of the stop-catalyzed EWB is now in direct conflict with data, since the light stops loops give unacceptably large shifts to the $h g g$ and $h \gamma \gamma$ couplings [20,21].

In this paper we take a different approach, following Ref. [27]. We assume that additional $\mathrm{TeV}$-scale physics beyond the MSSM provides a new tree-level contribution to the Higgs mass, reducing the need for large radiative corrections. There are many examples of such new physics, including (but not limited to) higher-dimensional F-terms $[25,26]$, or nondecoupling D-terms $[23,24]$. If all nonMSSM states introduced by such models are assumed to be sufficiently heavy, with masses around $1 \mathrm{TeV}$ or above, their effects at the $\sim 100 \mathrm{GeV}$ scale, relevant for both the EWPT and Higgs LHC phenomenology, can be parametrized as new hard-SUSY breaking terms in the Higgs potential. ${ }^{1}$ These effects can be parametrized by the generic two-Higgs doublet model (2HDM) potential:

$$
\begin{aligned}
V= & M_{u}^{2}\left|H_{u}\right|^{2}+M_{d}^{2}\left|H_{d}\right|^{2}+\left(b H_{u} \cdot H_{d}+\text { c.c. }\right) \\
& +\frac{\lambda_{1}}{4}\left|H_{u}\right|^{4}+\lambda_{2}\left|H_{u}\right|^{2}\left(H_{u} \cdot H_{d}+\text { c.c. }\right)+\lambda_{3}\left|H_{u}\right|^{2}\left|H_{d}\right|^{2} \\
& +\frac{\lambda_{4}}{2}\left(H_{u} \cdot H_{d}+\text { c.c. }\right)^{2}+\lambda_{5}\left|H_{u} \cdot H_{d}\right|^{2} \\
& +\lambda_{6}\left|H_{d}\right|^{2}\left(H_{u} \cdot H_{d}+\text { c.c. }\right)+\frac{\lambda_{7}}{4}\left|H_{d}\right|^{4} .
\end{aligned}
$$

In the MSSM, the coefficients are given by

$$
\begin{aligned}
& \lambda_{1}=\lambda_{7}=\frac{g^{2}+g^{\prime 2}}{2}, \quad \lambda_{3}=\frac{g^{2}-g^{\prime 2}}{4}, \\
& \lambda_{5}=-\frac{g^{2}}{2}, \quad \lambda_{2}=\lambda_{4}=\lambda_{6}=0 .
\end{aligned}
$$

Additional BSM physics can change these relationships. While such corrections explicitly break SUSY, one should note that the potential (2) is merely an effective description valid at energy scales $\sim 100 \mathrm{GeV}$, where SUSY is already broken. In the full UV theory, the corrections are generated by integrating out new physics at energy scales comparable to the soft SUSY-breaking scale, $\sim 1 \mathrm{TeV}$.

\footnotetext{
${ }^{1}$ Some SUSY models that lift the tree-level Higgs mass, e.g. nMSSM, introduce new states with masses $\sim 100 \mathrm{GeV}$. These states can have an important effect on the EWPT [17,18]. We will not consider such models here.
}

Not every term in the $2 \mathrm{HDM}$ potential can be easily generated by a UV-complete supersymmetric theory. For example, it difficult to see how any supersymmetric theory can generate nonzero coefficients $\lambda_{2}, \lambda_{4}$ and $\lambda_{6}$ at the tree level. The other coefficients can be generated either by new F-terms or by new D-terms at multi-TeV scale. However, only $\delta \lambda_{1}$ can give a substantial contribution to the SM-like Higgs mass without introducing new light states. ${ }^{2}$ For moderately large $\tan \beta$, a tree-level $125 \mathrm{GeV}$ Higgs mass requires $\delta \lambda_{1} \lesssim 0.1$, which can be easily accommodated in the low-energy effective field theory and UV-completed at a scale of $2 \ldots 3 \mathrm{TeV}$ [27]. Such hard-SUSY breaking term will be our basic assumption. Its presence completely removes the Higgs mass constraint on the stop masses and mixing. Apart from $\lambda_{1}$, all other $2 \mathrm{HDM}$ coefficients are assumed to be equal to the MSSM values.

Parenthetically we note that UV-complete theories which induce a necessary $\delta \lambda_{1}$ term will often also induce $\delta \lambda_{5}$ and $\delta \lambda_{7}$ of the same order of magnitude. We will disregard these terms in our analysis because they have no significant effect on the SM-like Higgs mass. Moreover, if the 2HDM is not in the full decoupling regime, these terms usually only make the fit of the Higgs couplings worse. Therefore the limit where $\delta \lambda_{5,7} \rightarrow 0$ can be regarded as the best possible case for the light stop SUSY scenario.

Two further simplifying assumptions will be made in our analysis. First, we assume that the 2HDM is in the decoupling regime, which for all practical purposes means $m_{A} \gtrsim 800 \mathrm{GeV}$. In this regime, the additional Higgs bosons beyond the SM-like $125 \mathrm{GeV}$ state are sufficiently heavy to have no effect at the EWPT critical temperature, $T_{c} \sim 100 \mathrm{GeV}^{3}$ This assumption is strongly motivated by the agreement of the LHC Higgs measurements with the SM: any deviation from these limits would only further strengthen the LHC constraints on the scenario in the large $\tan \beta$ regime of a supersymmetric theory. Second, we ignore the effect of all the superpartners, other than the stops, on the Higgs couplings and its thermal potential at $T \sim T_{c}$. This is motivated both by the nondiscovery of superpartners at the LHC, and by the fact that the Higgs coupling to stops is the strongest among its couplings to the superpartners. For example, while electroweak gauginos and Higgsinos may be present at a few-hundred GeV scale (and indeed may be needed to provide $C P$-violating phases in the EWB scenario), their effect on the Higgs properties is

\footnotetext{
${ }^{2}$ It has been shown in Ref. [27] that $\delta \lambda_{5}$ alone can also solve the problem of the Higgs mass. However, this solution either works for $\tan \beta \lesssim 3$, where there is no 1 st order EWPT, or requires $\delta \lambda_{5}>1$, which can probably be UV-completed only with new light states. Therefore we will disregard this option.

${ }^{3}$ We assume in this paper $m_{A} \approx m_{H} \approx m_{H^{ \pm}}$. While this is not true in generic $2 \mathrm{HDM}$, and an appreciable hierarchy between these masses can have peculiar consequences on the EWPT (see e.g. $[40,41])$, it is difficult to see how this hierarchy can be realized in a supersymmetric theory with no additional light states.
} 
subdominant to that of the stops. With these assumptions, both the EWPT and the collider constraints depend on just four unknown parameters $\left(m_{\tilde{t}_{1}}, m_{\tilde{t}_{2}}, \theta_{\tilde{t}}\right.$, and $\left.\tan \beta\right)$, enabling us to study the parameter space comprehensively and draw robust conclusions.

The spectrum required for the stop-catalyzed EWB would necessarily require some degree of fine-tuning. At one loop, stop masses receive a quadratically divergent QCD radiative correction, cut off by the gluino mass $m_{\tilde{g}}$. Complete naturalness requires $m_{\tilde{g}} \lesssim 2 m_{\tilde{t}}$ in the case of Majorana gluino, or $m_{\tilde{g}} \lesssim 4 m_{\tilde{t}}$ if the gluino is Dirac [42]. In either case, if the light stop is close to $100 \mathrm{GeV}$, gluinos must occur below $400 \mathrm{GeV}$; this possibility is comprehensively ruled out by the LHC data. Given the gluino bounds, a tuning of $\mathcal{O}(10-100)$ is required to accommodate such a light stop. Our philosophy in this paper is to not be concerned about this; we would like to know whether or not the stop-catalyzed EWB is in agreement with the data, regardless of fine-tuning issues.

\section{CONSTRAINTS FROM DIRECT SEARCHES AND HIGGS MEASUREMENTS}

First, we consider direct searches for stops at the LHC, where the light stop in the $100-120 \mathrm{GeV}$ mass range would be copiously produced. The sensitivity of the searches depends very strongly on the stop decay channels. There are two possible options, corresponding to R-parity conserving and R-parity violating scenarios. In the R-parity conserving framework a light stop can decay into $\tilde{\chi}^{0} b W^{(*)}$ via an off-shell top (and possibly also an off-shell $W$ ). Alternatively, in the mass range where 3-body stop decay is prohibited, one can expect the decay mode $\tilde{t} \rightarrow c \tilde{\chi}^{0}$ to compete with the four-body decay. All these decay channels were studied by ATLAS and CMS collaborations. The three- and four-body decays are searched for in monoleptonic channels $[43,44]$. While most of the parameter space is excluded, the bounds are discontinuous, and become much weaker or even completely disappear near the borderlines between three- and four-body decays of the stop. Moreover, none of these searches is optimized for the four-body decays, and the constraints in this region are weak. This leaves a very light stop in the $\sim 100 \mathrm{GeV}$ mass range still a viable possibility. The searches for the stop decays in the two-body $c \chi^{0}$ final state $[45,46]$ are more decisive and exclude the stops below the mass of $\sim 200 \mathrm{GeV}$ under the assumption that the branching ration (BR) into this mode is 1 . However, the exact BRs in this part of parameter space depend on the neutralino mixing angles, stop mixture, and possible flavor violation in the scalar sector beyond $\mathrm{MFV}^{4}$

\footnotetext{
${ }^{4}$ It has recently been claimed in Ref. [47] that the light stop scenario can be completely ruled out due to stoponium formation. It would be interesting to see whether a dedicated analysis by the experimental collaborations confirms this claim.
}

Another possibility is R-parity violation (RPV). In this case it is conceivable that the light stop is at the bottom of the spectrum and decays directly into SM states. Of course this possibility is excluded in the case of lepton-number violation. Searching for light stops in the baryon-number violating scenario, where the stop decays into two jets, with or without b-tag, is more challenging. However, recently CMS has excluded RPV stops below $200 \mathrm{GeV}$ [48], rendering this option irrelevant for the stop-catalyzed baryogenesis. Even stronger constraints have been obtained from an ATLAS boosted search [49], but they apply only for the b-tagged scenario.

To summarize, while direct constrains have already cornered the possibility of the light stops, the parameter space is not yet completely closed and the constraints are model-dependent. Therefore, we will now turn to analyzing indirect constraints, which are more robust.

An important set of constraints on the light stop scenario comes from the electroweak precision measurements. Split scalar multiplets at the electroweak scale, such as stops and bottoms in the presence of mixing, contribute to the $\mathrm{S}$ and $\mathrm{T}$ parameters [50]. But the strongest constraints currently come from the measurement of the Higgs properties at the LHC. In particular, loops of very light stops significantly modify the coupling of the Higgs to the photons and gluons. If the additional Higgs bosons of the 2HDM are not too heavy, Higgs-fermion couplings can also be modified. In the decoupling approximation, which is almost always true in the SUSY context, and for moderately large $\tan \beta$ this effect dominantly modifies the Higgs couplings to the down-type quarks and the taus [51].

Of course, the latter effect can be easily circumvented simply by decoupling the heavy Higgses. For example, if the heavy Higgs masses are around $800 \mathrm{GeV}$, we expect $\sim 3 \%$ correction to the $h \rightarrow b \bar{b}$ rate, much too small to be detected with the currently available data. On the other hand, corrections to the couplings to photons and gluons are much harder to address. The stop loop contribution to the $h \gamma \gamma$ and $h g g$ couplings both scale in the small mixing regime approximately as

$$
g_{\mathrm{hgg}} / g_{\mathrm{hgg}}^{\mathrm{SM}}-1 \approx \frac{1}{4}\left(\frac{m_{t}^{2}}{m_{\tilde{t}_{1}}^{2}}+\frac{m_{t}^{2}}{m_{\tilde{t}_{2}}^{2}}-\frac{m_{t}^{2} X_{t}^{2}}{m_{\tilde{t}_{1}}^{2} m_{\tilde{t}_{2}}^{2}}\right) .
$$

Without mixing, a $100 \mathrm{GeV}$ stop produces an $\mathcal{O}(100 \%)$ correction to the couplings, well beyond the 10\%-20\% level allowed by current LHC data. The only way to cancel this correction is to turn on the mixing, which effectively suppresses the coupling of the light stop to the Higgs. But this is in conflict with the requirements of the EWB scenario, which requires a near-maximal stop-Higgs coupling and therefore small mixing. As we will see in Sec. V, this tension cannot be reconciled with the current data. 
Before proceeding, let us note that the constraints from electroweak precision fits and the LHC Higgs measurements largely overlap, pointing to the same region in the stop parameter space [52-54]. In this region, called "funnel regime" or "blind spot" in the literature, the shift in the $h g g / h \gamma \gamma$ couplings and the stop contribution to the T parameter are both minimized. (The stop contribution to the $\mathrm{S}$ parameter is small and plays a subdominant role in the fits.) This occurs at approximately

$$
\sin (2 \theta) \approx \frac{2 m_{t}}{m_{\tilde{t}_{1}}-m_{\tilde{t}_{2}}} \quad \text { or } \quad X_{t} \approx m_{\tilde{t}_{1}}+m_{\tilde{t}_{2}}
$$

In the limit $m_{\tilde{t}_{1}} \ll m_{\tilde{t}_{2}}$, relevant for the stop-catalyzed EWB, these conditions simply mean that the light stop coupling to the Higgs vanishes, up to terms of order $\left(m_{\tilde{t}_{1}} / m_{\tilde{t}_{2}}\right)^{2}$ [see Eq. (9) below].

\section{ELECTROWEAK PHASE TRANSITION}

The viability of EWB requires that the EWPT be sufficiently strong, that is, that the rate for baryon number changing sphaleron transitions inside the broken phase, $\Gamma_{\mathrm{sph}}$, be slow enough to avoid washout of the baryon asymmetry. The rate $\Gamma_{\mathrm{sph}}$ is proportional to $\exp \left(-E_{\mathrm{sph}} / T\right)$, where $E_{\mathrm{sph}}$ is the sphaleron energy at temperature $T$. The transition proceeds when $T$ is below the bubble nucleation temperature, $T_{N}$, which is generally just below the critical temperature $T_{c}$. The larger the magnitude of $E_{\mathrm{sph}} / T_{N}$, the more effective will be the baryon asymmetry preservation in the broken phase.

In the context of perturbation theory, the computation of $\Gamma_{\text {sph }}$ entails considerable conceptual and theoretical challenges $[55,56]$. A particularly vexing one is maintenance of gauge-invariance. Loops containing gauge sector degrees of freedom (gauge bosons, unphysical scalars, FadeevPopov ghosts) introduce gauge-dependence into the finite temperature effective action, $S_{\text {eff }}(T)$. Obtaining a gaugeinvariant estimate of $\Gamma_{\text {sph }}$ at $T_{c}$ is possible [55], but doing so requires a level of care not typically followed in previous literature.

Here we adopt a strategy that can be appropriate for the MSSM and other scenarios wherein gauge degrees of freedom play a subdominant role in generating the barrier between the symmetric and broken vacua. Specifically, we truncate the one-loop effective potential $V_{\text {eff }}(T)$ at second order in the electroweak gauge couplings $g$ and $g^{\prime}$ while retaining while retaining terms to all orders in the top-quark Yukawa coupling, $y_{t}$. Doing so eliminates the gaugedependence that first arises at $\mathcal{O}\left(g^{3}\right)$ and that comes in tandem with the gauge-loop contribution to the barrier between the symmetric and broken-phase vacua. At the same time, it retains the gauge-invariant stop contributions to the barrier that enter first at $O\left(y_{t}^{3}\right)$ and that intuition tells us should dominate the phase transition dynamics.
This intuition is based on the stop contribution to the daisy resummation term in $V_{\text {eff }}(T)$ :

$$
\Delta V_{\text {daisy }}^{\tilde{t}}(T)=-\frac{2 N_{C} T}{12 \pi} \sum_{i=1,2}\left[M_{\tilde{t}_{i}}(h, T)^{3}-M_{\tilde{t}_{i}}(h)^{3}\right],
$$

where $N_{C}$ is the number of colors, " $h$ " generically denotes the vacuum expectation values of the two neutral doublet Higgses, $M_{\tilde{t}_{i}}(h)$ is the zero-temperature mass of stop eigenstate $t_{i}$ and $M_{\tilde{t}_{i}}(h, T)$ is the corresponding finite temperature mass. When the lighter eigenstate is essentially the right-handed stop, one has [13]

$$
M_{\tilde{t}_{1}}(h, T)^{2} \supset y_{t}^{2} h_{u}^{2}\left(1-\frac{X_{t}^{2}}{m_{Q}^{2}}\right)+m_{U}^{2}+\Pi_{\tilde{t}_{1}}(T),
$$

where $m_{Q}$ and $m_{U}$ are the left- and right-handed stop soft mass parameters, respectively, and $\Pi_{\tilde{t}_{1}}(T)$ is the one-loop thermal contribution to the stop mass-squared. Choosing $m_{U}^{2} \approx-\Pi_{\tilde{t}_{1}}(T)$ mitigates the screening of the stop contribution due to the daisy resummation. The resulting approximate $-T h_{u}^{3}$ term in the potential increases the barrier between broken and unbroken vacua, lowers $T_{c}$, and increases the ratio $E_{\mathrm{sph}} / T$ as needed for baryon number preservation [13]. For $T \sim 100 \mathrm{GeV}$, this choice leads to a lightest stop mass on the order of $100 \mathrm{GeV}$. Note that the coefficient of the stop-induced $-T h_{u}^{3}$ term is enhanced by $2 N_{C} y_{t}^{3}$. The gauge sector contributions, which are not included due to our truncation, carry no such enhancement.

The requirements for effective baryon number preservation follow from solving the sphaleron equations of motion and computing $E_{\mathrm{sph}}$. We observe that a consistent, nontrivial solution of these equations requires retaining gauge contributions to at least $\mathcal{O}\left(g^{2}\right)$ since the Higgs quartic self-couplings that enter the tree-level potential are $\mathcal{O}\left(g^{2}\right)$ as is the coupling between the gauge field and Higgs profile functions [57]. In the present setup, we formally retain all $\mathcal{O}\left(g^{2}\right)$ contributions, but include none at higher order in $g$ so as to maintain gauge invariance and consistency of the sphaleron equations of motion. In practice, for simplicity of numerical analysis we have not included the electroweak gauge boson contributions to the thermal masses that are also second order in $g$. We have estimated that doing so would result in shifts in the crucial quantity $\xi$, defined in Eq. (8), by no more than $10 \%$, leaving our conclusions unaffected.

The resulting baryon number preservation criterion can be expressed as a condition on the ratio [58]

$$
\xi \equiv \frac{v\left(T_{c}\right)}{T_{c}} \gtrsim 1
$$

where $v\left(T_{c}\right)$ is the value of $h(T)$ that minimizes $V_{\text {eff }}(T)$ at the critical temperature. As discussed in Ref. [55], there 
exist numerous sources of uncertainty in this condition, including the duration of the phase transition, the value of the baryon asymmetry at the start of the transition, the computation of the sphaleron fluctuation determinant, the origin of the unstable mode of the sphaleron, and neglected higher order loops $[56,59,60]$. Consequently, the precise numerical results should be taken with a grain of salt. Nevertheless, we believe that our qualitative conclusions will not be altered, even taking into account significant uncertainties associated with the use of perturbation theory to analyze the phase transition dynamics. To that end, we will show on our plots the contours of $\xi=0.5$ and $\xi=0$ (corresponding to the absence of a first order transition) to illustrate the potential impact of these uncertainties.

\section{RESULTS}

We performed a numerical scan over the fourdimensional parameter space outlined in Sec. II. For each point in the scan, we evaluated consistency with the experimental constraints by performing a fit to the LHC Higgs measurements, using the data set and the fitting procedure of Ref. [61], and the expressions for the coupling shifts from Ref. [52]. (For details of the fit, see Appendix A.) We also evaluated the constraints from EW precision measurements; however, we find these to be consistently weaker that the Higgs fit constraints.

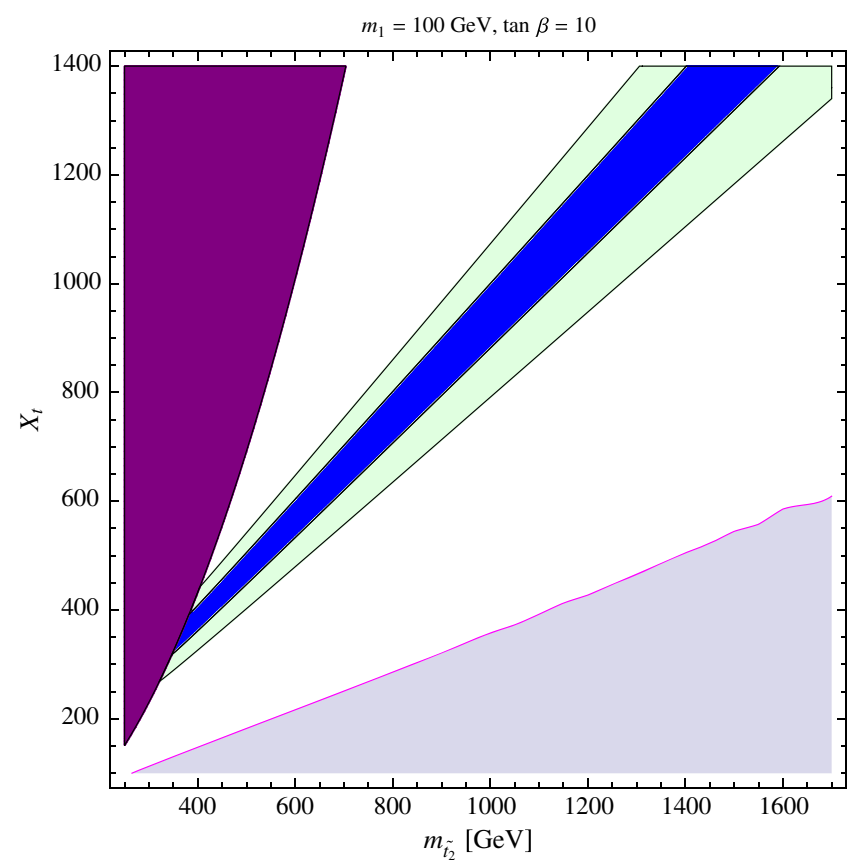

FIG. 1 (color online). Regions of stop parameter space allowed by the LHC Higgs measurements (blue-67\% CL and green95\% CL) vs. the domain where the stop-catalyzed EWB can potentially be viable $(\xi>0)$ in pink. Mass of the light stop is fixed at $100 \mathrm{GeV}$. The unphysical region (no solution for $\theta_{t}$ ) is shaded in purple.
Furthermore, for each point in the scan, we determined whether or not the EWPT is strongly 1st-order, using the procedure outlined in the previous section.

We find that the 1st-order phase transition requires a very light stop, $m_{\tilde{t}_{1}} \lesssim 110 \mathrm{GeV}$, independent of the other parameters. On the other hand, LEP-2 constraints imply $m_{\tilde{t}_{1}} \gtrsim 100 \mathrm{GeV}$, confining this parameter to a narrow band. Within this band, the Higgs fit constraints on the remaining parameters vary only slightly with $m_{\tilde{t}_{1}}$. In the plots below, we choose $m_{\tilde{t}_{1}}=100 \mathrm{GeV}$ as a representative value, but the picture that emerges from these plots is valid throughout the allowed range of $m_{\tilde{t}_{1}}$. Likewise, we fix $\tan \beta=10$ in the plots as a representative value. For larger $\tan \beta$ values our results stay almost independent of $\tan \beta$, while for lower $\tan \beta$ the EWPT becomes weaker while the Higgs constraints are largely unaffected. Again, the picture that emerges remains valid independent of $\tan \beta$.

The main results of our analysis are summarized in Figs. 1, 2 and 3. The conclusion is clear: there is no overlap between the parameter space regions allowed by the Higgs fit, and those consistent with a 1st-order EWPT within a perturbative calculation. Thus, the stop-catalyzed EWB scenario is no longer viable. Perhaps the clearest way to understand this result is provided by Fig. 1. Not surprisingly, the region of parameter space allowed by Higgs

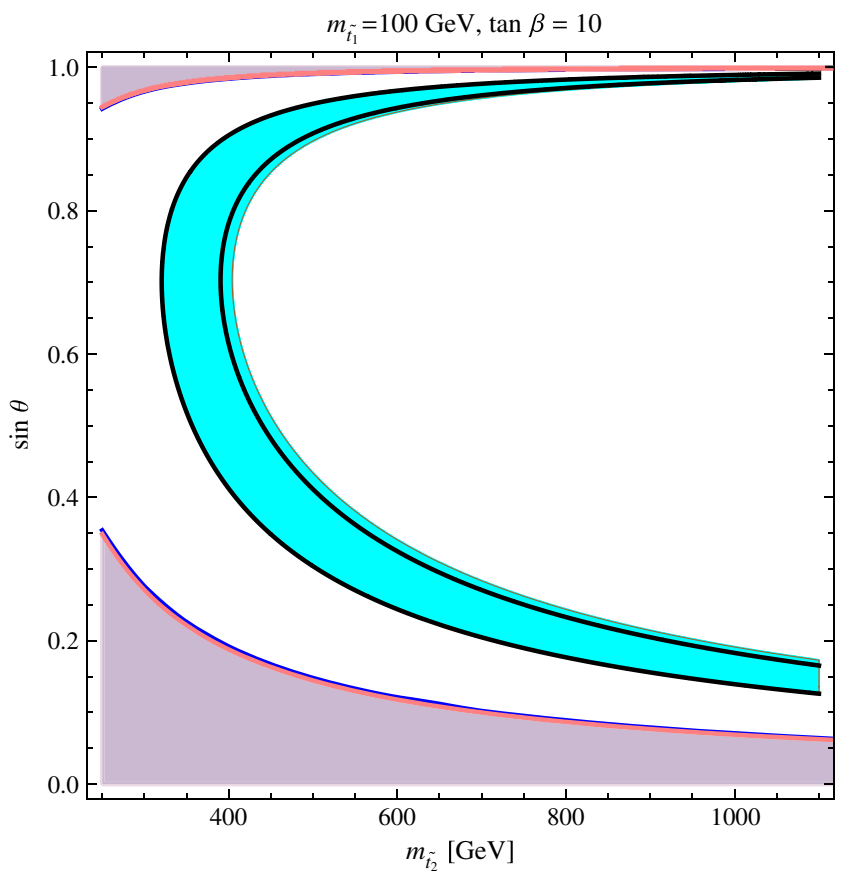

FIG. 2 (color online). Constraints and regions with a 1st-order EWPT in the $\left(m_{\tilde{t}_{2}}, \theta_{t}\right)$ plane, for the light stop mass $m_{\tilde{t}_{1}}=100 \mathrm{GeV}$. The region allowed by Higgs fits at 95\% CL is shaded in blue. The blue line shows the contour of $\xi=0$ and the red line shows the contour of $\xi=0.5$. The region between the black contours is allowed at the $95 \% \mathrm{CL}$ if a nonzero Higgs invisible width is included $\left(\epsilon_{\text {inv }}=0.1\right.$ using the definitions of [61]). 

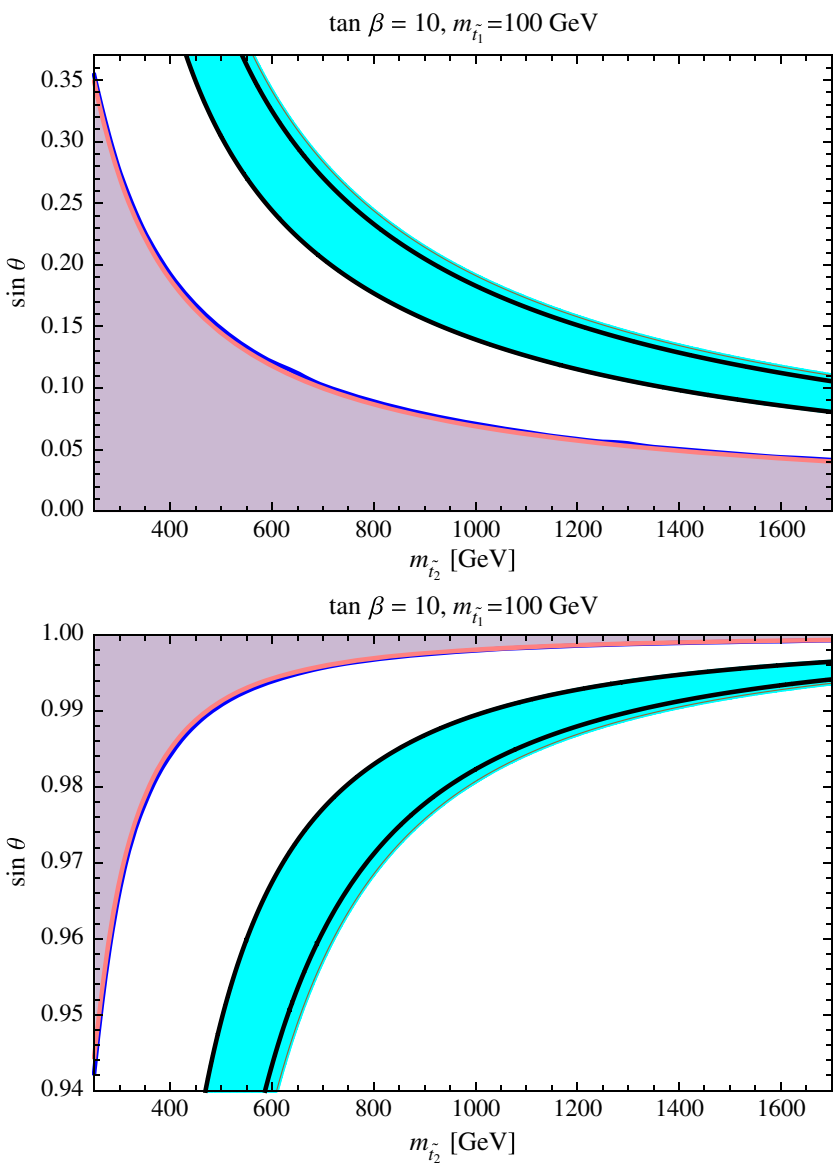

FIG. 3 (color online). Same as Fig. 2, zooming in on the regions where the light stop is mostly $\tilde{t}_{L}$ and $\tilde{t}_{R}$.

measurements is a band around the line $X_{t} \approx m_{\tilde{t}_{2}}$ : this follows directly from Eq. (4) in the limit $m_{\tilde{t}_{1}} \ll m_{\tilde{t}_{2}}$. The crucial observation is that along the contours of constant EWPT strength $\xi, X_{t}$ also scales linearly with $m_{\tilde{t}_{2}}$. This is related to the fact that the effective coupling between the lightest stop and the Higgs is given by

$$
\mathcal{L}_{\text {eff }}=y_{t}^{2}\left(1-\frac{X_{t}^{2}}{m_{\tilde{t}_{2}}^{2}-m_{\tilde{t}_{1}}^{2}}\right)|H|^{2}\left|\tilde{t}_{1}\right| .
$$

The thermal potential is determined almost exclusively by this effective coupling, so that constant- $\xi$ contours in the regime $m_{\tilde{t}_{1}} \ll m_{\tilde{t}_{2}}$ correspond to a fixed ratio $X_{t} / m_{\tilde{t}_{2}}$. Crucially, a 1st-order transition is only possible when the effective coupling is close to 1 ; specifically, $X_{t} / m_{\tilde{t}_{2}} \lesssim$ 0.3 is required, as can be seen in Fig. 1. This region does not overlap with the region $X_{t} / m_{\tilde{t}_{2}} \approx 1$ allowed by the Higgs fits, regardless of the value of $m_{\tilde{t}_{2}}$. Incidentally, this argument provides a clear understanding of the results of Ref. [20] regarding the MSSM, where $m_{\tilde{t}_{2}} \sim 100 \mathrm{TeV}$ is required by the $125 \mathrm{GeV}$ Higgs mass.
Another useful representation of the same results is shown in Figs. 2 and 3, where the $X_{t}$ parameter has been traded for the stop mixing angle $\theta_{t}$. These plots make it clear for a $100 \mathrm{GeV}$ light stop, the Higgs fits imply a tight relationship between $\theta_{t}$ and $m_{\tilde{t}_{2}}$, which unfortunately is incompatible with the small-mixing regime required by the stop-catalyzed EWB.

Now we address an important potential caveat to the above argument. The Higgs coupling fit presented above is performed under the assumption of no new Higgs decay channels beyond those present in the SM. Lifting this assumption may be expected to relax the Higgs fit constraints. In particular, the strong constraint on the gluon coupling comes from the agreement of the inferred production cross section in the dominant $g g \rightarrow h$ channel with the SM. If the Higgs has a new decay channel, a production cross section in excess of the SM value can be accommodated without changing the observed event rates in any of the channels. In the stop-catalyzed EWB scenario, it is quite natural for the Higgs to have an appreciable decay width into light neutralino pairs, resulting in an invisible final state. It was argued in Ref. [28] that this may revive the stop-catalyzed EWB scenario, even within the MSSM.

However, since Ref. [28] appeared, dedicated experimental searches for Higgs invisible decays have been performed by both ATLAS and CMS, which strongly constrain this possibility $[62,63]$. Reference [61] combined two these measurements (both of which had downward fluctuations) and estimated the allowed Higgs invisible branching ratio as $-0.18 \pm 0.31$, of course perfectly consistent with zero. The bound on $h \gamma \gamma$ coupling has also been improved significantly, so that the gluon coupling does not dominate the fit as strongly as in the early analyses of the LHC Higgs data. Due to both these factors, turning on the Higgs invisible width no longer improves the overall Higgs couplings fit, and does not relax the constraints in the "right" direction for the stop-catalyzed EWB scenario. We explicitly illustrate this point in Figs. 2 and 3, which shows that with the current data, allowing for the Higgs invisible width does not improve the fit.

\section{CONCLUSIONS}

While stop-catalyzed EWB is a theoretically attractive scenario, it has been known for some time that the LHC measurements of the Higgs mass and rates rule it out in the case of the MSSM. In this paper, we extended the analysis to supersymmetric models with nonminimal contributions to the Higgs potential. Such contributions can lift the treelevel Higgs mass above the MSSM bound, thus eliminating one of the most stringent MSSM constraints on the stop sector parameters. One might hope that the newly expanded parameter space would include regions compatible with a stop-catalyzed EWB. We showed that this is unfortunately not the case. The reason for this is simple: the stopcatalyzed EWB requires a light stop $\left(m_{\tilde{t}_{1}} \sim 100 \mathrm{GeV}\right)$ with 
near-maximal coupling to the Higgs, since otherwise the effects of stop loops on the Higgs thermal potential are not large enough to trigger a strong 1st-order electroweak phase transition. On the other hand, such a light stop is compatible with the LHC constraints on the $h g g$ and $h \gamma \gamma$ couplings only if its coupling to the Higgs is suppressed, and is far from maximal. We quantified these requirements and found no overlap between the parameter space regions with viable stop-catalyzed EWB and those with Higgs couplings compatible with the LHC data. The conclusion holds even in the presence of non-SM invisible Higgs decay channels such as $h \rightarrow \tilde{\chi}^{0} \tilde{\chi}^{0}$.

An important limitation of our analysis is the assumption that no additional scalars beyond a single SM-like Higgs participate in the electroweak phase transition. This assumption can be violated in nonminimal supersymmetric models with extended Higgs sectors. For example, in the NMSSM-like scenario, an additional gauge-singlet scalar field can be active during the phase transition, leading to novel possibilities such as a two-step phase transition. It has been shown that a strong 1st-order electroweak phase transition remains a viable possibility in this class of models [16-18]. In these cases, the potential barrier necessary for the strong 1st-order transition typically arises from the tree-level multifield scalar potential, rather than thermal loop effects, making them quite distinct from the stop-catalyzed scenario we focused on here. Still, these models demonstrate that even though the stop-catalyzed EWB no longer appears viable, other options for successful EWB exist in the supersymmetric context.

The analysis of this paper provides an illustration of the power of the Higgs data collected by the LHC to shed light on the electroweak phase transition, an important event in the history of the Universe about which we currently have no direct information. The current 10\%-20\% accuracy of the Higgs coupling measurements is already sufficient to rule out one of the most popular scenarios with a strong 1storder phase transition. Many other models (both SUSY and non-SUSY) that allow for a strong 1st-order phase transition predict more subtle deviations from the SM, and testing these models will require increasing the precision of the Higgs coupling measurements to the $1 \%$ level and beyond, as well as measuring the Higgs cubic self-coupling [32,64-66]. The proposed electron-positron Higgs factories and a $100 \mathrm{TeV}$ proton-proton collider will be needed to comprehensively probe the viability of a strong 1st-order EWPT, and hence of electroweak baryogenesis.

\section{ACKNOWLEDGMENTS}

We are grateful to Roberto Franceschini, Michelangelo Mangano and Veronica Sanz for useful discussions. The work of A.K. was accomplished at the Aspen Center for Physics, which is supported by National Science Foundation grant PHY-1066293. The research of A. K. and M. J. R. M. was also supported by the Munich Institute for Astro- and Particle Physics (MIAPP) of the DFG cluster of excellence "Origin and Structure of the Universe". M. P. is supported by the U.S. National Science Foundation through Grant No. PHY-1316222. M. J. R. M. and P. W. were supported in part by U.S. Department of Energy Contract No. DE-SC0011095.

\section{APPENDIX: DETAILS OF THE HIGGS COUPLINGS FIT}

Constraints on the stop sector parameters were derived from a fit to the Higgs couplings measured at the LHC. In this Appendix we describe the fitting procedure in more detail. Since the procedure closely follows the prescription of Ref. [61], one can view this Appendix as a short executive summary of this reference.

The Higgs couplings taken into account in the fit are: $h b \bar{b}, h g g, h \rightarrow$ inv, $h W W, h Z Z, h \gamma \gamma$ and $h \tau \tau$. In the main part of our analysis, which assumes that the 2HDM is in the decoupling regime, the only deviations are in $h \gamma \gamma$ and $h g g$ and, in the case when Higgs decay to a neutralino pair is allowed, in $h \rightarrow$ inv. The rest of the couplings only become important when we explicitly check the effects of $2 \mathrm{HDM}$ outside of the decoupling regime, which necessarily triggers deviations in the couplings to the down type sector fermions and to the lesser extend to the gauge bosons.

First, we slightly simplify the "proper" fit, by assuming that all error bars in the LHC measurements are Gaussian (which does not lead to a significant loss of information). Second, we assume that all deviations from the SMpredicted values are small, namely $r_{i}=1+\epsilon_{i}$, where $r_{i}$ 's are defined as

$$
\begin{aligned}
\mathcal{L}_{\text {higgs }}= & r_{\psi} \frac{m_{\psi}}{v} h \bar{\psi} \gamma \psi+r_{Z} \frac{M_{Z}^{2}}{v} h Z^{\mu} Z_{\mu}+r_{W} \frac{2 m_{W}^{2}}{v} h W_{\mu} W^{\mu} \\
& +r_{\gamma} c_{\mathrm{SM}}^{\gamma \gamma} \frac{\alpha}{\pi v} h F_{\mu \nu}^{2}+r_{g} c_{\mathrm{SM}}^{g g} \frac{\alpha_{s}}{\pi v} h G_{\mu \nu}^{2},
\end{aligned}
$$

and $\epsilon_{i} \ll 1$. For the invisible rate, which is expected to vanish in the $\mathrm{SM}$, we assume $\operatorname{BR}(h \rightarrow$ inv $)=\epsilon_{\text {inv }}$. To estimate the measured deviation of the $\gamma \gamma$ coupling from the SM we use the results of ATLAS [67] and CMS [68]. By combining these results we estimate $\epsilon_{\gamma}=0.00 \pm 0.14$ (of course we quote $1 \sigma$ uncertainties). The coupling $h g g$ is extracted from all the exclusive (gluon fusion) decay modes, namely $\gamma \gamma$ [67,68], $Z Z^{*}[69,70], W W^{*}$ [71,72], and to the lesser extent from the fermionic channels. The average is $\epsilon_{g}=-0.13 \pm 0.20$. For the extraction of the bounds on the deviations of the other couplings, which are less important in our fit, the reader is again referred to Ref. [61]. In the basis $\epsilon_{i}=\left(\epsilon_{b}, \epsilon_{g}, \epsilon_{\mathrm{inv}}, \epsilon_{W}, \epsilon_{Z}, \epsilon_{\gamma}, \epsilon_{\tau}\right)$, the correlation matrix between the different $\epsilon_{i}$ 's, based on the theoretical calculation, is 


$$
\rho=\left(\begin{array}{ccccccc}
1 & 0.70 & 0.04 & 0.52 & 0.38 & 0.58 & 0.59 \\
0.70 & 1 & 0.43 & 0.38 & 0.11 & 0.40 & 0.52 \\
0.04 & 0.43 & 1 & 0.46 & 0.13 & 0.40 & 0.34 \\
0.52 & 0.38 & 0.46 & 1 & 0.44 & 0.63 & 0.45 \\
0.38 & 0.11 & 0.13 & 0.44 & 1 & 0.42 & 0.33 \\
0.58 & 0.40 & 0.40 & 0.63 & 0.42 & 1 & 0.54 \\
0.59 & 0.52 & 0.34 & 0.45 & 0.33 & 0.54 & 1
\end{array}\right) .
$$

For completeness we also quote here the allowed ranges of the other $\epsilon$ 's, as calculated by [61]:

$$
\epsilon_{b}=-0.19 \pm 0.28
$$

$$
\begin{gathered}
\epsilon_{\mathrm{inv}}=-0.22 \pm 0.20, \\
\epsilon_{W}=-0.2 \pm 0.13, \\
\epsilon_{Z}=0.00 \pm 0.10, \\
\epsilon_{\tau}=-0.03 \pm 0.17 .
\end{gathered}
$$

Again, these channels were important in the fit only when we considered the nondecoupling limit of the 2HDM and/or the possibility of an invisible decay of the Higgs to light neutralinos.
[1] D. E. Morrissey and M. J. Ramsey-Musolf, Electroweak baryogenesis, New J. Phys. 14, 125003 (2012).

[2] A. Sakharov, Violation of $C P$ invariance, c asymmetry, and baryon asymmetry of the universe, Pis'ma Zh. Eksp. Teor. Fiz. 5, 32 (1967).

[3] M. Gurtler, E.-M. Ilgenfritz, and A. Schiller, Where the electroweak phase transition ends, Phys. Rev. D 56, 3888 (1997).

[4] M. Laine and K. Rummukainen, What's new with the electroweak phase transition?, Nucl. Phys. B, Proc. Suppl. 73, 180 (1999).

[5] F. Csikor, Z. Fodor, and J. Heitger, Endpoint of the Hot Electroweak Phase Transition, Phys. Rev. Lett. 82, 21 (1999).

[6] Y. Aoki, F. Csikor, Z. Fodor, and A. Ukawa, The endpoint of the first order phase transition of the SU(2) gauge Higgs model on a four-dimensional isotropic lattice, Phys. Rev. D 60, 013001 (1999).

[7] M. B. Gavela, P. Hernandez, J. Orloff, and O. Pene, Standard model $C P$ violation and baryon asymmetry, Mod. Phys. Lett. A 09, 795 (1994).

[8] P. Huet and E. Sather, Electroweak baryogenesis and standard model $C P$ violation, Phys. Rev. D 51, 379 (1995).

[9] M. B. Gavela, P. Hernandez, J. Orloff, O. Pene, and C. Quimbay, Standard model $C P$ violation and baryon asymmetry. Part 2: Finite temperature, Nucl. Phys. B430, 382 (1994).

[10] Y. Li, S. Profumo, and M. Ramsey-Musolf, Bino-driven electroweak baryogenesis with highly suppressed electric dipole moments, Phys. Lett. B 673, 95 (2009).

[11] V. Cirigliano, Y. Li, S. Profumo, and M. J. Ramsey-Musolf, MSSM baryogenesis and electric dipole moments: An update on the phenomenology, J. High Energy Phys. 01 (2010) 002.

[12] J. Kozaczuk, S. Profumo, M. J. Ramsey-Musolf, and C. L. Wainwright, Supersymmetric electroweak baryogenesis via resonant sfermion sources, Phys. Rev. D 86, 096001 (2012).
[13] M. Carena, M. Quiros, and C. E. M. Wagner, Opening the window for electroweak baryogenesis, Phys. Lett. B 380, 81 (1996).

[14] D. Delepine, J. M. Gerard, R. Gonzalez Felipe, and J. Weyers, A light stop and electroweak baryogenesis, Phys. Lett. B 386, 183 (1996).

[15] M. Carena, G. Nardini, M. Quiros, and C. Wagner, The baryogenesis window in the MSSM, Nucl. Phys. B812, 243 (2009).

[16] M. Pietroni, The electroweak phase transition in a nonminimal supersymmetric model, Nucl. Phys. B402, 27 (1993).

[17] A. Menon, D. E. Morrissey, and C. E. M. Wagner, Electroweak baryogenesis and dark matter in the nMSSM, Phys. Rev. D 70, 035005 (2004).

[18] S. J. Huber, T. Konstandin, T. Prokopec, and M. G. Schmidt, Electroweak phase transition and baryogenesis in the nMSSM, Nucl. Phys. B757, 172 (2006).

[19] J. Engel, M. J. Ramsey-Musolf, and U. van Kolck, Electric dipole moments of nucleons, nuclei, and atoms: The standard model and beyond, Prog. Part. Nucl. Phys. 71, 21 (2013).

[20] D. Curtin, P. Jaiswal, and P. Meade, Excluding electroweak baryogenesis in the MSSM, J. High Energy Phys. 08 (2012) 005.

[21] T. Cohen, D. E. Morrissey, and A. Pierce, Electroweak baryogenesis and Higgs signatures, Phys. Rev. D 86, 013009 (2012).

[22] A. Menon and D. E. Morrissey, Higgs boson signatures of MSSM electroweak baryogenesis, Phys. Rev. D 79, 115020 (2009).

[23] P. Batra, A. Delgado, D. E. Kaplan, and T. M. P. Tait, The Higgs mass bound in gauge extensions of the minimal supersymmetric standard model, J. High Energy Phys. 02 (2004) 043.

[24] A. Maloney, A. Pierce, and J. G. Wacker, D-terms, unification, and the Higgs mass, J. High Energy Phys. 06 (2006) 034. 
[25] M. Dine, N. Seiberg, and S. Thomas, Higgs physics as a window beyond the MSSM (BMSSM), Phys. Rev. D 76, 095004 (2007).

[26] X. Lu, H. Murayama, J. T. Ruderman, and K. Tobioka, A Natural Higgs Mass in Supersymmetry from Nondecoupling Effects, Phys. Rev. Lett. 112, 191803 (2014).

[27] A. Katz, M. Reece, and A. Sajjad, Naturalness, $b \rightarrow s \gamma$, and SUSY heavy Higgses, J. High Energy Phys. 10 (2014) 102.

[28] M. Carena, G. Nardini, M. Quiros, and C. E. Wagner, MSSM electroweak baryogenesis and LHC data, J. High Energy Phys. 02 (2013) 001.

[29] S. Profumo, M. J. Ramsey-Musolf, and G. Shaughnessy, Singlet Higgs phenomenology and the electroweak phase transition, J. High Energy Phys. 08 (2007) 010.

[30] H. H. Patel and M. J. Ramsey-Musolf, Stepping into electroweak symmetry breaking: Phase transitions and Higgs phenomenology, Phys. Rev. D 88, 035013 (2013).

[31] H. H. Patel, M. J. Ramsey-Musolf, and M. B. Wise, Color breaking in the early universe, Phys. Rev. D 88, 015003 (2013).

[32] S. Profumo, M. J. Ramsey-Musolf, C. L. Wainwright, and P. Winslow, Singlet-catalyzed electroweak phase transitions and precision Higgs boson studies, Phys. Rev. D 91, 035018 (2015).

[33] M. Jiang, L. Bian, W. Huang, and J. Shu, Impact of a complex singlet: From dark matter to baryogenesis, arXiv:1502.07574.

[34] N. Blinov, J. Kozaczuk, D. E. Morrissey, and C. Tamarit, Electroweak baryogenesis from exotic electroweak symmetry breaking, Phys. Rev. D 92, 035012 (2015).

[35] S. Inoue, G. Ovanesyan, and M. J. Ramsey-Musolf, Twostep electroweak baryogenesis, arXiv:1508.05404.

[36] S. Heinemeyer, W. Hollik, and G. Weiglein, Precise prediction for the mass of the lightest Higgs boson in the MSSM, Phys. Lett. B 440, 296 (1998).

[37] S. Heinemeyer, W. Hollik, and G. Weiglein, The Masses of the neutral $C P$-even Higgs bosons in the MSSM: Accurate analysis at the two loop level, The European Physical Journal C 9, 343 (1999).

[38] M. Carena, H. E. Haber, S. Heinemeyer, W. Hollik, C. E. M. Wagner, and G. Weiglein, Reconciling the two loop diagrammatic and effective field theory computations of the mass of the lightest $C P$-even Higgs boson in the MSSM, Nucl. Phys. B580, 29 (2000).

[39] P. Draper, P. Meade, M. Reece, and D. Shih, Implications of a $125 \mathrm{GeV}$ Higgs for the MSSM and low-scale SUSY breaking, Phys. Rev. D 85, 095007 (2012).

[40] G. C. Dorsch, S. J. Huber, and J. M. No, A strong electroweak phase transition in the 2HDM after LHC8, J. High Energy Phys. 10 (2013) 029.

[41] G. C. Dorsch, S. J. Huber, K. Mimasu, and J. M. No, Echoes of the Electroweak Phase Transition: Discovering a Second Higgs Doublet through $A_{0} \rightarrow Z H_{0}$, Phys. Rev. Lett. 113, 211802 (2014).

[42] C. Brust, A. Katz, S. Lawrence, and R. Sundrum, SUSY, the third generation and the LHC, J. High Energy Phys. 03 (2012) 103.

[43] G. Aad et al. (ATLAS), Search for top squark pair production in final states with one isolated lepton, jets, and missing transverse momentum in $\sqrt{s}=8 \mathrm{TeV} p p$ collisions with the ATLAS detector, J. High Energy Phys. 11 (2014) 118.
[44] CERN Technical Report No. CMS-PAS-SUS-13-011, 2013, URL http://cds.cern.ch/record/1547550.

[45] CERN Technical Report No. CMS-PAS-SUS-13-009, 2014, URL https://cds.cern.ch/record/1644584.

[46] G. Aad et al. (ATLAS), Search for pair-produced thirdgeneration squarks decaying via charm quarks or in compressed supersymmetric scenarios in $p p$ collisions at $\sqrt{s}=$ $8 \mathrm{TeV}$ with the ATLAS detector, Phys. Rev. D 90, 052008 (2014).

[47] B. Batell and S. Jung, Probing light stops with stoponium, J. High Energy Phys. 07 (2015) 061.

[48] V. Khachatryan et al. (CMS), Search for pair-produced resonances decaying to jet pairs in protonproton collisions at $\sqrt{s}=8 \mathrm{TeV}$, Phys. Lett. B 747, 98 (2015).

[49] CERN Technical Report No. ATLAS-CONF-2015-026, 2015, URL http://cds.cern.ch/record/2037653.

[50] M. Drees, K. Hagiwara, and A. Yamada, Process independent radiative corrections in the minimal supersymmetric standard model, Phys. Rev. D 45, 1725 (1992).

[51] K. Blum and R. T. D’Agnolo, 2 Higgs or not 2 Higgs, Phys. Lett. B 714, 66 (2012).

[52] J. R. Espinosa, C. Grojean, V. Sanz, and M. Trott, NSUSY fits, J. High Energy Phys. 12 (2012) 077.

[53] N. Craig, M. Farina, M. McCullough, and M. Perelstein, Precision Higgsstrahlung as a probe of new physics, J. High Energy Phys. 03 (2015) 146.

[54] J. Fan, M. Reece, and L.-T. Wang, Precision natural SUSY at CEPC, FCC-ee, and ILC, J. High Energy Phys. 08 (2015) 152.

[55] H. H. Patel and M. J. Ramsey-Musolf, Baryon washout, electroweak phase transition, and perturbation theory, J. High Energy Phys. 07 (2011) 029.

[56] T. Cohen and A. Pierce, Electroweak baryogenesis and colored scalars, Phys. Rev. D 85, 033006 (2012).

[57] J. M. Moreno, D. H. Oaknin, and M. Quiros, Sphalerons in the MSSM, Nucl. Phys. B483, 267 (1997).

[58] M. Quiros, Finite temperature field theory and phase transitions, arXiv:hep-ph/9901312; URL http://alice.cern .ch/format/showfull?sysnb $=0302087$.

[59] J. Espinosa, Dominant two loop corrections to the MSSM finite temperature effective potential, Nucl. Phys. B475, 273 (1996).

[60] M. S. Carena, M. Quiros, and C. Wagner, Electroweak baryogenesis and Higgs and stop searches at LEP and the Tevatron, Nucl. Phys. B524, 3 (1998).

[61] P. P. Giardino, K. Kannike, I. Masina, M. Raidal, and A. Strumia, The universal Higgs fit, J. High Energy Phys. 05 (2014) 046.

[62] CERN Technical Report No. ATLAS-CONF-2013-011, 2013, URL http://cds.cern.ch/record/1523696.

[63] CERN Technical Report No. CMS-PAS-HIG-13-018, 2013, URL http://cds.cern.ch/record/1561758.

[64] A. Noble and M. Perelstein, Higgs self-coupling as a probe of electroweak phase transition, Phys. Rev. D 78, 063518 (2008).

[65] A. Katz and M. Perelstein, Higgs couplings and electroweak phase transition, J. High Energy Phys. 07 (2014) 108.

[66] D. Curtin, P. Meade, and C.-T. Yu, Testing electroweak baryogenesis with future colliders, J. High Energy Phys. 11 (2014) 127. 
[67] CERN Technical Report No. ATLAS-CONF-2013-012, 2013, URL http://cds.cern.ch/record/1523698.

[68] V. Khachatryan et al. (CMS), Observation of the diphoton decay of the Higgs boson and measurement of its properties, Eur. Phys. J. C 74, 3076 (2014).

[69] G. Aad et al. (ATLAS), Measurements of Higgs boson production and couplings in diboson final states with the ATLAS detector at the LHC, Phys. Lett. B 726, 88 (2013); 734, 406(E) (2014).
[70] S. Chatrchyan et al. (CMS), Measurement of the properties of a Higgs boson in the four-lepton final state, Phys. Rev. D 89, 092007 (2014).

[71] CERN Technical Report No. ATLAS-CONF-2013-030, 2013, URL http://cds.cern.ch/record/1527126.

[72] S. Chatrchyan et al. (CMS), Measurement of Higgs boson production and properties in the WW decay channel with leptonic final states, J. High Energy Phys. 01 (2014) 096. 